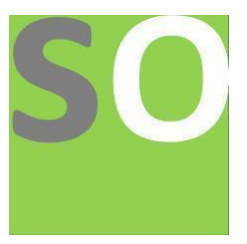

Article title: Leisure Studies on Program Output With Delay Relaxation.

Authors: Frank Appiah[1]

Affiliations: King's Engineers Group[1]

Orcid ids: 0000-0001-6732-9503[1]

Contact e-mail: appiahnsiahfrank@gmail.com

License information: This work has been published open access under Creative Commons Attribution License $\mathrm{http}: / / c r e a t i v e c o m m o n s . o r g / l i c e n s e s / b y / 4.0 /$, which permits unrestricted use, distribution, and reproduction in any medium, provided the original work is properly cited. Conditions, terms of use and publishing policy can be found at https://www.scienceopen.com/.

Preprint statement: This article is a preprint and has not been peer-reviewed, under consideration and submitted to ScienceOpen Preprints for open peer review.

DOI: 10.14293/S2199-1006.1.SOR-.PPZRZDZ.v1

Preprint first posted online: 16 April 2021

Keywords: Leisure, Relaxation, keyboard, Screen, Delay 


\title{
Leisure Studies on Program Output With Delay Relaxation.
}

\section{Prof Frank Appiah} appiahnsiahfrank@yahoo.com 14.04.2021

\begin{abstract}
Interactive computing environments consisting of screen and keyboard provides a means to relax and enjoy the program output. Leisurely, ways to slow and relax program execution is delved with system calls like delay execution, synthesis execution and file management execution. The leisure time can be the exact delay time used in slowly the chances of output activity.
\end{abstract}

Keywords. Leisure, keyboard, screen, interactive computing, delay relaxation.

\section{Introduction}

Till now, thus far we have emphasized interactive computing environments consisting of a video screen and keyboard. In that mode, a program from time to time requests data of the user and from time to time produces output, typically intermixing input and output.

Computing is sometimes done in what it is called batch mode. In batch mode, the program and all the data are fed into the computer at one time, and the output is not available until after the program has run to completion.

Data stored in memory in units called files. Off-line data is read into programs without the user entering the data during program execution. This process is to delay execution time. Programs written to read offline data differ from the kinds of interactive programs that seem to handle input and output. Online data is used by programs without file input. A stored program that handles input and data along the program instructions to an output device. This is online data processing. There is no enjoyment in whether you do online or offline processing but there is delay relaxation in waiting on 
the system operation. It can slow applications to create a leisurely account for the user. Too many in programs do we find too fast unleisure program execution to the very end without much interaction. Interactive computing environment for leisure purposes delve on these properties :

- Slow system activity to counter fast program

- Leisure time as delay relaxation on interaction.

- Synthesis activity to auditory invoked response activity.

- Color data activity on coloring output screen to user enjoyment.

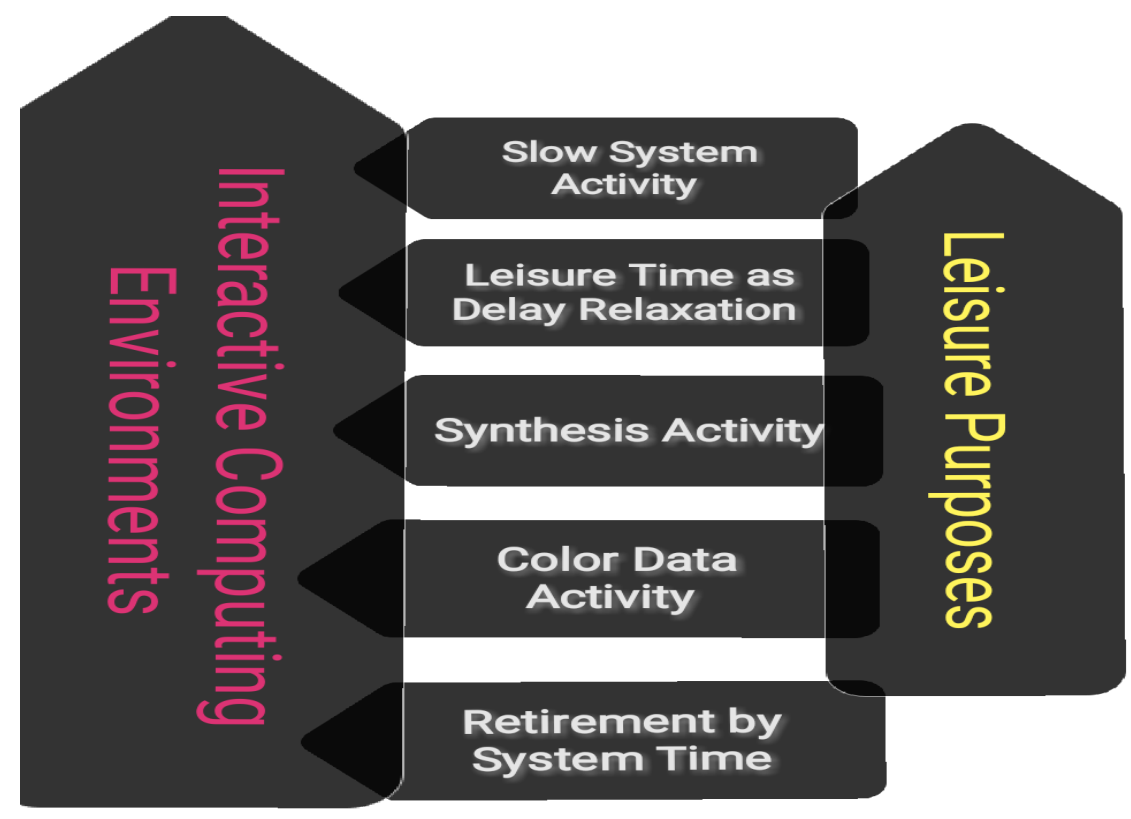

- Retirement by system time with interactive keyboard inputs.

The concern here is that the developer has a fast program that reads data and to the very end brings results to output. Delving on leisure purposes is to create fun and enjoyment at the same time before the program ends in execution. Using the first property, which is slow system activity to counter fast program. With this property, file inputting can be sorted to read data into the leisure purpose program. The second property is to initiate a leisure time as delay relaxation on interaction. For sometime in $\mathrm{t}$ delay time or leisure time, program waits for (1)some input to be made(system delay is in use) (2) user to read text on screen in relax mode instead of fast mode and (3) attend to an earlier information from program and continue later on execution to run.

A code in Pascal will look like this: 


\section{infopowe.pas $X$}

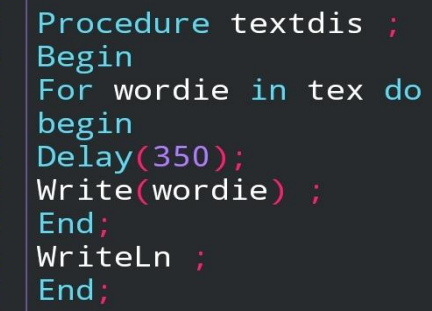

This procedure textdis will show a word in tex list one by one after the delay time or leisure time equals 350 milliseconds.

On the third, synthesis activity is required in leisure purposed interactive computing environments (LePICE) in order to bring voice relaxation to the user. The invocation of auditory response is a perception and sensation of hearing. Hearing reduces the fast interaction as you stop hear definitely on sound and that is good or perfect leisure activity in LePICE.

A code in Pascal will look like this:

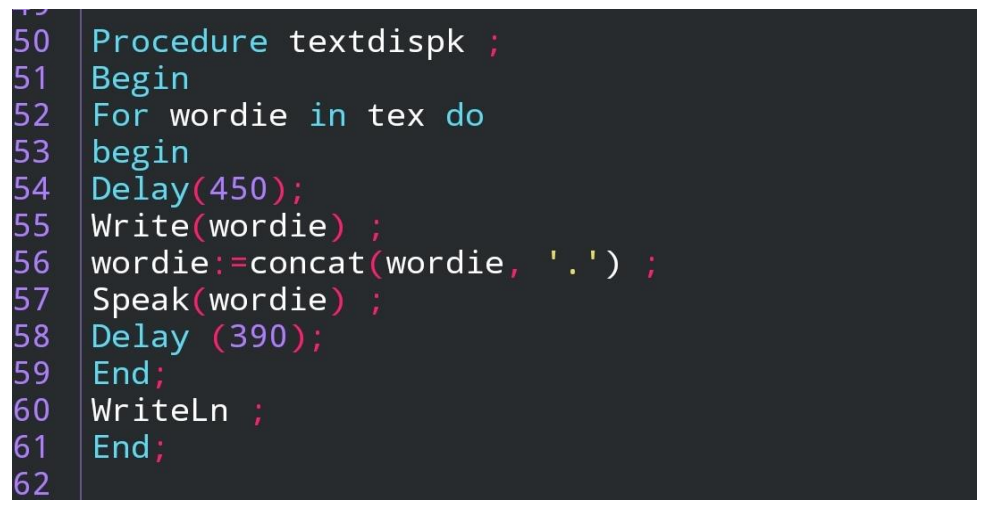

The procedure textdispk, will read data in a list structure tex then hold on in leisure time of about 450 milliseconds before it writes a word to the output device. After the write process, it then speaks each word in the list/array. There is a relaxation delay of about $350 \mathrm{~ms}$ before the next voice on word. In color data activity, the part of the body for perceiving and sensing is the eye. Pleasant experience with the eye is leisurely accurate in dealing relaxant LePICE. Good sight is worth enjoying in LePICE for leisure purposes.

A code in Pascal will look like this: 


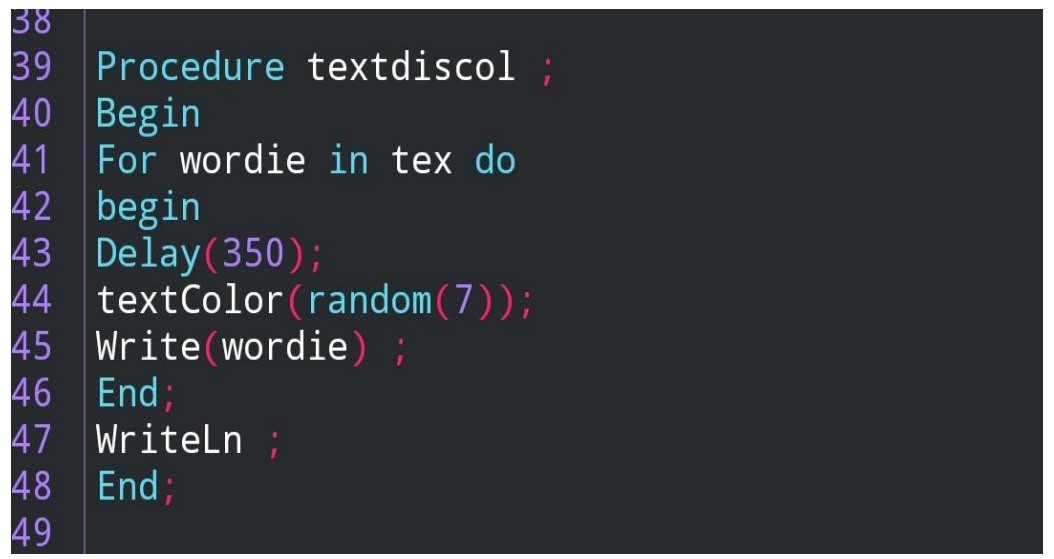

The procedure textdiscol, on random input from 0 to 7 generates a color index for text coloring and finally writes the word in the tex array after a relaxation delay of $350 \mathrm{~ms}$. There is an initial delay about the same value of $350 \mathrm{~ms}$. Lastly, it is retirement by System time on interrupt. This is retirement because it will hang permanently until it receives input on the keyboard. This system time retires on interrupt to create LePICE. The blinking cursor shows the retirement indicator to accept input from the user or continue retiring.

The pascal code is

Read(varname);

I will now look at some delay time samples:

\begin{tabular}{|l|l|l|l|}
\hline Delay Time/ms & Number of Write & Number of Speak & Used Function \\
\hline 2500 & 1 & 1 & Textdis \\
\hline 6000 & 4 & 5 & Textdis \\
\hline 3000 & 0 & 1 & Textdiscol \\
\hline 3600 & 1 & 1 & Not Applicable \\
\hline 3700 & 1 & 1 & Not Applicable \\
\hline 1500 & 1 & 0 & Not Applicable \\
\hline 4500 & 2 & 1 & Not Applicable \\
\hline 17890 & 1 & 1 & Not Applicable \\
\hline 3500 & 1 & 1 & Not Applicable \\
\hline
\end{tabular}




\begin{tabular}{|c|c|c|c|}
\hline 3800 & 0 & 1 & textdiscol \\
\hline 14578 & 1 & 1 & Not Applicable \\
\hline 1880 & 1 & 0 & Not Applicable \\
\hline 3000 & 0 & 1 & Not Applicable \\
\hline 3000 & 2 & 1 & Not Applicable \\
\hline 2500 & 1 & 1 & Not Applicable \\
\hline 2590 & 1 & 1 & Not Applicable \\
\hline 15890 & 0 & 1 & Textdispk \\
\hline 5680 & 3 & 0 & Not Applicable \\
\hline 2700 & 0 & 1 & Not applicable \\
\hline 3000 & 2 & 1 & Not Applicable \\
\hline 1500 & 1 & 0 & Not Applicable \\
\hline 3000 & 1 & 1 & Not Applicable \\
\hline 1200 & 0 & 0 & Not Applicable \\
\hline 26000 & 1 & 1 & Not Applicable \\
\hline 25090 & 2 & 2 & Not Applicable \\
\hline 18000 & 0 & 1 & Not Applicable \\
\hline 19000 & 0 & 1 & Not Applicable \\
\hline 20000 & 1 & 1 & Not Applicable \\
\hline 20000 & 1 & 1 & Not Applicable \\
\hline 20000 & 1 & 1 & Not Applicable e \\
\hline 19000 & 1 & 1 & Not Applicable \\
\hline 3000 & 0 & 1 & Textdispk \\
\hline 20000 & 1 & 1 & Not Applicable \\
\hline
\end{tabular}




\section{Conclusion}

This work did describe a concept about leisure purposed interactive computing environments. For shorthand is LePICE. Five properties of LePICE were discussed. Three code constructs in Pascal were looked at in syntax. Finally, a table of Sample delay relaxation or leisure time was made.

\section{Further Reading}

1. Anderson, H. A., \& Sargent, R. G. (1974). Investigation into scheduling for an interactive computing system. IBM journal of research and development, 18(2), 125-137.

2. Wegner, P. (1998). Interactive foundations of computing. Theoretical computer science, 192(2), 315-351.

3. Anderson Jr, H. A., \& Sargent, R. G. (1972). A statistical evaluation of the scheduler of an experimental interactive computing system. In Statistical Computer Performance Evaluation (pp. 73-98). Academic Press.

4. Wegner, P., \& Goldin, D. (1999). Mathematical models of interactive computing. 